\title{
Navigating Evidence Based Practice: A Guide for Nursing Students
}

\section{Lissa Gagnon *}

\author{
School of Nursing, Laurentian University, Canada
}

\begin{abstract}
Nurses have, as part of their professional standards, an obligation to be able to explain and support their clinical decisions on the basis of evidence-based practice (EBP). For example, in Ontario, the regulatory body entitled the College of Nurses of Ontario [1] makes reference to EBP as the first listed indicator for each of two of its practice standards. Evidence-based practice has become a foundational and influential concept in both clinical and educational settings. Health care providers such as nursing students, registered nurses, and nurse educators all have roles to ensure that EBP is respected in the delivery of patient care.

For nursing students, EBP may not be an immediate concern. However, their obligation to master the evidence that underlies effective nursing evolves into an responsibility to guide their practice, as they become nurses. Nurses and nurse educators have additional obligations to stay current and to develop a clear understanding of the nature and quality of the evidence that exists. The purpose of this paper is twofold: First, to provide a theoretical discussion for nursing students on EBP's history and its impact on nursing practice; and second, to focus on how students and nurses' attitudes, education, and both individual and organizational barriers influence EBP utilization in practice. This paper addresses the academic discourse on how frequently and effectively EBP is actually incorporated into nursing praxis as a means to enhance quality advancement in nursing pedagogy. Nursing students, nurses, and nurse educators should be aware of the complex state EBP exists within nursing and how challenging it can be to accurately implement. The origins of EBP in nursing were largely adopted from the medical field as they embraced new standards to ensure quality practice. This history aided in increasing the relevance of nursing as a professional practice, yet produced some challenges nurses and nursing students still contend with today. Scholars have attempted to understand the complex set of factors, which contribute to effective EBP utilization. Several of these issues continue to be studied within the nursing profession, such as positive nurses' attitudes towards EBP, and research serving to increase the rate of utilization. Other factors act as barriers to EBP utilization with the profession. Gaps in EBP practitioner knowledge or conflicts with policy are two examples identified to be barriers to effective EPB utilization. Contemporary issues and potential future directions must also be considered in order to address the challenges of operating EBP in both educational and clinical settings.
\end{abstract}

Keywords

Evidence-based practice, Evidence-informed practice, Nursing students, Nursing education, Attitudes and barriers of evidence-based practice, Impact of evidence-based practice

\section{Introduction}

Evidence-based practice is a concept, which weaves through not only the nursing profession, yet the medical as well as other health care fields. It has become part of the professional practice guidelines for nurses and an expectation as segment of the greater health care system. Most nursing faculty, students, and professionals would agree that EBP is a positive force improving the quality of care. However, the reality is that there are complexities to the process of utilizing evidence to guiding practice. Even the definition of EBP requires consideration.
A purpose of this paper is to provide a guide for nursing students to help understand the concept and mobiliza-

*Corresponding author: Lissa Gagnon, School of Nursing, Laurentian University, Ramsey Lake Road, Sudbury, On Canada, P3E 2C6, Canada, Tel: 705-675-1151 ext. 3705 (voice), Fax: 705-675-4861

Accepted: September 22, 2018;

Published online: September 24, 2018

Citation: Gagnon L (2018) Navigating Evidence Based Practice: A Guide for Nursing Students. J Nurs Pract 2(2):73-80

Copyright: (C) 2018 Gagnon L. This is an open-access article distributed under the terms of the Creative Commons Attribution License, which permits unrestricted use, distribution, and reproduction in any medium, provided the original author and source are credited. 
tion of EBP in the nursing profession as well as its influencing factors. Nursing students are introduced to the topic of EBP early in their education and a clear understanding of the concept can be helpful throughout education, clinical, and professional life. Engaging early with EBP will help students understand the myriad of factors involved and help prepare them for its utilization in a variety of situations.

The first step to the goal of effectively navigating EBP is to review its origins and evolving definition. Once the ideas of EBP are embedded in their historical context, it will be easier to consider how it relates to other factors of practice. This paper will subsequently focus on the influence of attitudes towards EBP, personal and organizational barriers to EBP utilization, as well as address the question of how nursing research relates.

Evidence-based practice can be defined in its most fundamental form as the "conscientious use of current best evidence in making decisions about patient care [2]". Boswell and Cannon define EBP as "a process of using confirmed evidence, decision-making, and nursing expertise to guide the delivery of holistic patient care ( $\mathrm{p}$. 6)" [3]. Regardless of various definitions, EBP has always reflected the core goal of improving the quality of care and patient outcomes by incorporating the latest research evidence into practice. This disparity may account for one reason the terms EBP and research utilization are used interchangeably in the nursing literature. However, many nurse researchers contend they are not synonymous [2,4-8]. For example, Estabrooks, et al. [4], recognizing the confusion around EBP, describes research utilization as the use of research findings. She contends that EBP is a broad concept that encompasses more than the integration of research evidence into practice.

There has been some confusion amongst nursing scholars with regards to what domains EBP encompasses. For example, early definitions were simple and emphasized the link of using research studies to guide decisions made in clinical situations. This definition made it almost synonymous with research utilization, which is the act of using research to make a positive change in the nursing environment. Research utilization was often used in reference to a single study [2]. Many authors contended EBP incorporates health care providers' personal expertise as well as personal assessments and patient preferences. Some scholars suggested these broadened definitions might compromise the core rigor, which is assumed to be present with EBP [2,5-8]. These researchers also debate about how much personal expertise and patient preference should be allowed into the practice of EBP.

In some contexts, the concept of EBP has evolved into "evidence informed decision-making" or "evidence informed practice" (EIP). The Canadian Nurses Association position statement [9] on evidence based decision-making and nursing practice defines EIP as "a continuous interactive process involving the explicit, conscientious and judicious consideration of the best available evidence to provide care" (p.1). This refined definition acknowledges the increased complexity and differing roles in which nurses have taken responsibility. For example, promoting community health and considering the cost effectiveness of care are acknowledged as important components in this latest definition. The direct translation of evidence into practice is rarely simple, and EIP ensures that the use of evidence is understood to be an evolving exercise.

EIP has been comprehended as the use of best clinical evidence to guide nurses in making sound clinical decisions based not only on research, but also on clinical experience, client preferences, and cost effective treatment [2,5-8]. Similarly, the concept of critical thinking in nursing literature has undergone philosophical transformations over time. For example Meherali, Profetto-McGrath and Paul [10] emphasize that critical thinking is also seen as a strategy to ensure that the evidence is relevant to the clinical situation as experienced by undergraduate nursing students [2-8]. This paper shall focus on describing EIP once the historical development of EBP has been discussed. The rationale for this sequencing is to ensure that nursing students focus on the latest definition of the term, as it will be more helpful upon graduation.

Polit and Beck [11] elaborate that, in light of rapidly evolving practices in the nursing profession, nurses are required to inform and modernize their nursing skills by referring to extant research or, increasingly, by conducting or participating in research to inform their practice. These authors further point toward the following future directions of EIP in nursing by indicating that nurses will continue to be encouraged to engage in EIP and utilize research data effectively. For further evaluation, a greater emphasis should be placed on systematic reviews, more commonly be conducted in educational facilities, community, and clinical settings. It is imperative for health care that nursing education continues to adapt curricula to meet the challenges of an ever changing nursing environment.

\section{Background Literature}

\section{Origins of evidence-based practice}

Evidence-based practice originated in the 1970's as an outgrowth of evidence-based medicine and physicians' decisions to enhance practice with scientific evidence $[2,4]$. There was pressure from academia as well as the public to consolidate and communicate the latest 
scientific findings in order to improve patient outcomes. This large-scale effort required the collaboration of many health care fields including nursing. One of the results was the creation of a global database of systematic reviews known as the Cochrane Collaboration.

The Cochrane Collaboration served as a base for professionals from several disciplines to share and evaluate research. Strength of research is a vital component in mobilizing effective EBP $[2,4]$. Research, using the scientific method, could now be evaluated for quality and aggregated to provide guidance for clinical practice. Part of the agenda of the Cochrane Collaboration was that, in principle, the establishment of a common core of evaluative principles would in turn guide researchers to use better methods and more careful research designs thus improving quality.

Researchers suggest that EBP in nursing arose from evidence-based medicine [2,4,12-14]. In particular the Cochrane Collaboration initiatives spurred nursing to adapt and begin using research in order to justify interventions and produce measureable patient outcomes. According to the Canadian Association of Schools of Nursing [15], new skills including research appraisals and focus on research domains such as methodology would force the nursing educational system to incorporate these concepts.

The next challenge was incorporating these new research findings into practical settings. At the time of the Cochrane Collaboration publication, the nursing field was also experimenting with research utilization projects such as the Conduct and Utilization of Research in Nursing as a means to promote the application of research findings into the clinical setting [4]. One of the consequences of the decision to rely on research evidence to guide practice, and the subsequent increase in nursing research, is that nursing as a field suddenly found itself acting more autonomously than it had before when it comes to making changes and improving practice. The transition was not simple partly because it required both development of research and critical expertise within the nursing world but also because nursing practice is normally cooperative with other fields.

As early as 1975, Ketefian [16] began addressing the gap between knowledge gained from research and the use of that knowledge in practice, as this was among the priorities for the nursing profession. If nursing aims to utilize research findings to improve clinical decision-making, then individual and organizational barriers need to be identified, understood, and addressed within the health care system $[2,11,16]$.

Since the 1970's, further EBP discourse focused on two relevant aspects; nurses' attitudes of research, and the barriers of EBP utilization. For example, empirical studies have shown nurses' attitudes to be important factors that contribute to EBP in nursing [17-23]. In addition, empirical work examines nurses' and students' perceptions of the barriers related to the application of EBP [24].

In summary, nursing adopted EBP from doctors and other professionals; this was heavily influenced by the Cochrane Collaboration. Because the evidence about nursing practice is largely in the hands of nurse researchers, nursing became more independent in improving and altering its practice and education. Nursing scholars began to examine the process of translating knowledge obtained from research into clinical practice. Nursing curricula evolved to incorporate research appraisal and its application to the clinical environment. The value placed on scientific method as a set of standards for research led to the embedding of EBP or EIP as a responsibility for all those in the nursing profession.

\section{Factors influencing evidence informed practice utilization}

The next section shall help nursing students understand various factors, which influence EIP in the practical environment. Nursing students should be aware of how EIP will realistically present itself in nursing environments. Every nursing situation, which utilizes EIP, is unique and is subject to its own influences and challenges. Nursing students who understand these influential factors will be better equipped to engage with EIP both in the classroom and work environment. The factors, which shall be focused on, are nursing attitudes and barriers to EIP implementation.

There are a complex variety of factors, which operate at any given time to determine how effective EIP is incorporated into education and practice. These factors include attitudes toward EBP. Attitudes begin with nursing students and the culture they receive from nursing educators. Perceived barriers to implementation of knowledge are also important to comprehend. Finally, the level of nursing education and the way in which EIP is incorporated into curricula play a large role in how effective it is used within the nursing profession.

Nursing student and nurse attitudes towards EIP are known to be a significant factor in determining its adherence. As Lacey suggests, "attitude alone is a powerful predictor of utilization" (p.992). Theoretically, nursing students and professionals who have a positive attitude towards EIP and research are more likely to engage with it and effectively utilize it $[23,25]$. Negative attitudes likely make people less willing to engage with complex activities such as EIP. Therefore, attitudes towards EIP are important to study at all levels of nursing education as well as in practice. 
Nursing educators should encourage students to have a positive attitude regarding EIP because this is more likely to lead to students utilizing it effectively in clinical settings for positive patient outcomes [26,27]. Researchers have found that nurses who believe research is important and useful in providing information tend to make more sound clinical decisions $[17,18,28]$. They also suggest that nurses are interested in research for improving their work environment and patient care $[17,19,23,29]$. Positive attitudes coupled with positive changes, which result from applying EIP, make the relationship between attitudes and EIP worth investigating [12].

Numerous nurse researchers have studied nurses' attitudes of research in the clinical setting [17-21,23,2537]. The researchers indicated that nurses have an overall positive attitude towards research regarding personal growth and guidance of nursing practice [20,21,31$33,36,38]$. It is worth noting that not all attitudes towards research and EIP were positive. Some researchers such as Marsh and Brown reported that educational levels did not influence nurses' perceptions of research. Others found that educational background influenced nurses' attitudes towards research [29,35], for example, nurses who were baccalaureate of nursing prepared had a higher interest in research compared with diploma preparation.

Contemporary literature has echoed similar positive attitudes when asking nurses of various positions about EBP [25-27,30]. Clinical nurses continue to have positive attitudes towards EBP and this remains to be a strong indicator of a nurses' use of EBP. However, other studies such as from White-Williams and colleagues [25] suggest that stronger indicators of a nurse applying EBP effectively are knowledge of EBP, higher education level, and skill with the topic. One author suggested that factors such as attitude and knowledge may not have as great an influence as originally thought as it pertains to effective use of EBP by nurses and nursing students [26]. Overall, nurses continue to have a positive attitude despite the challenges associated with the implementation of EBP.

Ultimately, nurses' attitudes and EIP appear to have some connection. It is suggested by most research that positive attitudes towards EIP helps predict its usage in the future. The initial formation of attitude towards research and EBP begins during post secondary nursing education. Therefore, understanding nurses' attitudes is important as a curriculum is being evaluated. Utilizing EIP is a responsibility for nurses and nursing students and is mandated by the Canadian Nurses Association. This obligation means that nursing students must learn about EIP despite challenges or barriers, which may make it challenging.

There are a large variety of factors preventing nurses from transferring research findings into practice; many of which have been identified in the nursing literature. Researchers have consistently recognized this issue and have surveyed nurses who work in clinical settings to determine their views on barriers encountered when trying to use research findings in practice $[20,23,24,39,40-52]$. The diverse barrier types may be due to the broad range of settings nurses practice, ranging from community to hospital settings.

The main findings of these studies assisted in understanding the reasons that research is not being transformed into EBP. Lack of support from the organization was a common barrier reported in the literature $[21,24,36,39,41,42,45,48,53]$. Workload and lack of authority to change practice are examples of the organization's lack of support regarding implementation. Another common reported barrier relates to the presentation of credibility in research journals $[24,37,52,53]$. The authors suggest that more user-friendly publications are essential. For example, these researchers suggest that explaining the meaning of statistical results and identifying implications for practice would help nurses understand the findings and facilitate the development of research-based practice. In addition, lack of time has been noted as the greatest barrier $[19,21,24,35,36,38,42,44,48,49,51,53]$. This lack of time represents both time to read research and time to implement findings into the present practice.

Recent studies appear to support many of the past studies citing that clinical nurses perceive lack of knowledge related to research utilization as well as lack of organizational support $[27,30,54]$. Other barriers were cited to be lack of knowledge, experience, time and mentors for EIP. One study may contradict the long-standing assumption that barriers are a powerful predictor of whether nurses will implement EIP in the field [55]. The relationships between barriers and nurse knowledge, attitude and actual EIP utilization are not well studied and there may be other variables to address when educating new nurses as well as nurses in the field. These realities leave several gaps to be addressed in future nursing research and education.

With regards to nursing students, a positive and early introduction to EIP may aid in alleviating some barriers and ease the use of EIP in the field. However, new graduate nurses will most likely work for an organization, which operates under the guidance of policy. These policies are extremely powerful in influencing how care is delivered. Nurses are often limited to how EIP can be implemented due to the constraints placed by policy and the pressure for nurses to perform using methods that ensure that their responsibilities and license are protected. Barriers and challenges are often unique to different settings and work environments. 


\section{Discussion}

The use of research findings in nursing has contributed to the literature for over 20 years [21,45]. A number of researchers, however, propose that the application of research findings in practice remains low $[50,51,56]$. Mulhall supports this concern and reports that only a modest number of nurses apply research in their practice. These findings support the need to determine the indicator of evidence-based research being implemented in the nursing pedagogy and clinical settings.

Countless numbers of breakthroughs in treatments, policies, and approaches to nursing care have ensured that nursing education remains vital in the greater health care system. In fact, given the importance of EIP, nurse educators should be prepared to provide students with an understanding and opportunities of engagement with EIP and its applicability in contemporary practice. For example, while nursing students and nurses use available evidence, the application and outcomes may vary depending on patient preferences, concerns, and values. Therefore, those who judge EIP as "cookbook nursing" may be failing to recognize the flexibility of the function of the many components of this concept [57]. Supporting EIP is a reflection of a current mandate in the nursing profession, as this problem-solving approach has resulted in improved care and better patient outcomes [12].

Despite the fact that there has been a paradigm shift in adapting EBP in clinical care, the literature indicates that there remains much progress for its implementation in daily practice and as a component of nursing education $[2,58]$. To date, nurses' attitudes of research and their perceived barriers of knowledge transfer remains a professional concern for nursing pedagogy. Other factors such as the nurses' ability to think critically may influence how effectively nurses implement EBP. Nurse educators have long focused on critical thinking as a major goal within nursing education [10]. Continuing analysis of the relationships between nurses' attitudes, barriers, and skills as they relate to EBP while incorporating other elements such as critical thinking may allow the nursing education system to better prepare nurses to engage with EIP.

In addition to nursing students and nurse ability to utilize EIP, there are concerns about the types of evidence being utilized by nurses. The Cochrane Collaboration was largely based upon the medical system, which primarily accepts quantitative paradigms as the best evidence. Nurse researchers are known for having a large body of research in the qualitative paradigm. This mismatch of research types often makes it more difficult for professionals outside of the nursing profession to accept qualitative evidence. Quantitative evidence may be seen as superior to qualitative in some circles due to its commonality and ease of use as evidence. Qualitative research is not valued as highly in other professions and can be a cause of tension when it is brought forward as evidence.

The predominance of empirical literature identified in this paper that examines nurses' attitudes of research and nurses' perceptions on barriers to EIP is quantitative in nature. This does not mean that qualitative research is not valuable. Qualitative research is strong in examining experiences that are often complex and do not always translate into a cause and effect intervention for practice. A review of qualitative paradigms of these bodies of literature may complement the trends of EIP's evolution. Future qualitative research could focus on abetting nurses to address the implications of the use of EIP from classrooms to clinical settings. More research is needed to effectively translate qualitative evidence into practice.

Over the years nurse researchers have studied nurses' attitudes toward research in the clinical setting and the concept of EBP [17,29]. In general, these studies indicated that nurses are interested in research for improving their work environment and patient care. As well, they have an overall positive attitude towards research, believing it is important and useful in providing information to make evidence-based clinical decision-making. Rizzuto and colleagues surveyed over 1200 practicing nurses on research activities. Findings from this survey indicated nurses agree that research and practice should be linked to ensure quality care. The awareness of this association is vital for nursing's professional growth. A number of researchers, however, report that despite these positive attitudes, limited research findings are transferred into the clinical setting $[2,50,51,56,59]$. The overall historic positive attitude towards EBP and research theoretically bodes well for increased utilization. However, with the abundance of other factors influencing EBP utilization nurse attitudes may not be as of a strong indicator as originally thought and other lesser-studied factors may have a greater influence. For example, the policies at health organizations and barriers could have a greater influence in the modern context. All of these factors can be incorporated into nursing curricula to raise awareness about these factors and how they will manifest outside the classroom.

One question that has not yet been addressed is "how much evidence is enough to warrant change?" No one argues that if sufficient evidence is presented changes should be made to improve practice. The point of sufficient evidence is complex because the point of accepting evidence strong enough for change varies between professionals and organizations. There is also the challenge of evidence providing only marginal improvements to 
practice. These changes may never be implemented because the change is not worth the effort or resources. The ability to critically evaluate evidence becomes more valuable because the value of the evidence itself is not the only factor. The ease of incorporation and financial burden of a proposed change must also be analyzed. Overcoming these obstacles presents another barrier to EIP use.

Several researchers have also surveyed nurses who work in clinical settings to determine their views on barriers encountered when trying to transfer research into practice [2,59]. Some of the common barriers identified within this body of work include lack of time, as well as support from health care administration. These realities reflect the need to determine the reasons that research is not being transferred into practice. Successful research implementation may require more than dissemination alone, suggesting that organizational strategy can help promote EIP. Looking at addressing these barriers is a complex endeavor involving many players including nurses, health organizations and nurse educators. Optimally, nurse educators would strive to ensure that these barriers are highlighted so that nurses and health organizations understand their significance and potentially seek to implement solutions and evaluate their effectiveness.

Nursing care environments can be complex with a wide variety of different health care professionals with different expertise. Expertise could be seen as a barrier to nurses EIP implementation because other practitioners operate using their own sets of guidelines. This makes it difficult for nurses who may have found applicable research or a new way to accomplish a task to enact lasting changes. Nurses may be dismissed due to perceived inexperience and because others such as doctors dismiss claims due to the self belief in their expertise. Nursing students should be aware that convincing other health care practitioners or even other nurses to adapt due to new evidence could be challenging or frustrating. However, this should not deter students from advocating for themselves and pushing to create environments which are more accepting to new evidence being incorporated.

Despite the existing barriers of EIP in workplace environments, it is noteworthy that nurses mostly view research favorably in terms of their role and responsibilities with patient care. As mentoring nurses, this acuity is valuable for the development of nursing students in terms of professional socialization. However, the discourse remains whether EIP is the responsibility of individuals or organizations, or a combination thereof. Theoretically, while nursing students enter the clinical environment and critically analyze existing policies in order to assess its use of evidence, which, if any, ramifications exist when nursing staff may be unable to support the students' initiatives? Subsequently, does the implemen- tation of EIP become the nurses' responsibility to lobby for support when the policies exclude evidence? Furthermore, will students question if the support solely rests with health care administrators? In the end, who will drive EBP and address the need for evidence in clinical policies and procedures?

Several studies have demonstrated that focused educational interventions have proven to be effective in addressing knowledge, skill and attitude gaps related to EIP in both nurses and nursing students $[26,60]$. Educational intervention has been shown to aid front line nurses equipping them with confidence, new skills and understanding of EIP. It does not take a stretch of the imagination to assume that similar focused information sessions given to nursing students could produce similar effects. In addition, there are other variables, which may be studied in order to provide a broader understanding of nursing relation to EIP. For example, the influences of the interdisciplinary teams in health care settings as well as the influence of nursing EIP leadership in these settings are not well understood and may bring greater perspective to the discourse of effective nursing EIP utilization.

\section{Conclusion}

While a vast majority of nurses and nursing students understand the importance and broad definition of EBP, there are still shortcomings in its utilization. Nurse educators and researchers must continue to broaden understanding on the nurses' relation to EIP to help identify strengths and weaknesses of different nursing levels in various environments ranging from school to clinical. Nurse educators and clinical leaders must seek ways to focus EIP information and deliver content where student nursescan be confident and engaged with its usage. This need includes focusing on influencing factors such as attitudes, barriers, skills, and policy. Much of this approach begins with nursing students in the classroom. Exposing nursing students to the historical context of EPB and evolution of EIP will increase their knowledge and upsurge its visibility within the nursing profession both in classrooms and practice. Nursing students' acquired knowledge and exposure to EIP should aid in advancing nurse pedagogy as well as EIP use in an ever-changing nursing environment. Nurses have progressed with the use of EIP, yet there exist further challenges that require attention at all levels of the nursing profession.

\section{Acknowledgment}

The authors wish to acknowledge Dr. Ginette Roberge for her contributions.

\section{References}

1. College of Nurses of Ontario (2002) Professional standards. Revised 2002. 
Citation: Gagnon L (2018) Navigating Evidence Based Practice: A Guide for Nursing Students. J Nurs Pract 2(2):73-80

2. Melnyk B, Fineout-Overholt E (2011) Evidence-based practice in nursing \& healthcare. WoltersKluer/Lippincott Williams \& Wilkins, New York.

3. Boswell C, Cannon S (2014) Introduction to nursing research: Incorporating evidence-based practice. ( $3^{\text {rd }}$ edn), Jones and Bartlett, Sudbury,

4. Estabrooks CA (1998) Will evidence-based nursing practice make practice perfect? Canadian Journal of Nursing Research 30: 15-36.

5. NadalinPenno L (2008) Understanding the motivation of nurses towards the continued use of evidence-based practice in a tertiary clinical setting: An application of plannedbehaviour theory. (Unpublished Master's Thesis). University of Ottawa, Ottawa, ON.

6. Poirier $P(2013)$ Nursing-led management of side effects of radiation: evidence-based recommendations for practice. Nursing: Research and Reviews 3: 47-57.

7. Schneider Z, Whitehead D (2012) Nursing and midwifery research: Methods and critical appraisal for evidence-based practice. Elsevier Australia.

8. Stetler CB, Ritchie JA, Rycroft-Malone J, et al. (2009) Institutionalizing evidence-based practice: an organizational case study using a model of strategic change. Implementation Science 4: 1-19.

9. Canadian Nurses Association (2010) Position Statement "Evidence-informed decision making and nursing practice".

10. Meherali SM, Profetto-Mc Grath J, Pail P (2015) Nursing students critical thinking and research utilization. Quality Advancement in Nursing Education 1: 1-15.

11. Polit DF, Beck C (2014) Essentials of research: appraising evidence for nursing practice. ( $8^{\text {th }}$ edn), Lippincot Williams \& Wilkins, China.

12. Melnyk B, Fineout-Overholt E (2005) Making the case for evidence-based practice. In BM.

13. Mowinski Jennings B, Loan LA (2001) Misconceptions among nurses about evidence-based practice. J Nurs Scholarsh 33: 121-127.

14. Mulhall A (1998) Nursing, research, and the evidence. Evidence-Based Nursing 1: 4-6.

15. Canadian Association of Schools of Nursing (2015) National nursing education framework final report.

16. Ketefian S (1975) Application of selected nursing research findings into nursing practice: A pilot study. Nursing Research 24: 89-92.

17. Alcock D, Carroll G, Goodman M (1990) Staff nurses' perceptions of factors influencing their role in research. Can $\mathrm{J}$ Nurs Res 22: 7-18.

18. Berggren AC (1996) Swedish midwives' awareness of, attitudes to and use of selected research findings. J Adv Nurs 23: $462-470$

19. Bostrom AC, Malnight M, Mac Dougall J, et al. (1989) Staff nurses' attitudes toward nursing research: A descriptive survey. J Adv Nurs 14: 915-922.

20. Champion VL, Leach A (1989) Variables related to research utilization in nursing: An empirical investigation. $J$ Adv Nurs 14: 705-710.
21. Lacey EA (1994) Research utilization in nursing practice - a pilot study. J Adv Nurs 19: 987-995.

22. Rempusheski VM (1991) Incorporating research role and practice role. Applied Nursing Research 4: 46-48.

23. Yates $P$, Baker D, Barrett L, et al. (2002) Cancer nursing research in Queensland, Australia: Barriers, priorities, and strategies for progress. Cancer Nursing 25: 167-180.

24. Funk SG, Champagne MT, Wiese RA, et al. (1991) Barriers to using research findings in practice: The clinicians perspective. Appl Nurs Res 4: 90-95.

25. White-Williams C, Patrician P, Fazeli $P$, et al. (2013) Use, Knowledge, and Attitudes Toward Evidence-Based Practice Among Nursing Staff. J Contin Educ Nurs 44: 246-254.

26. Leach MJ, Hofmeyer A, Bobridge A (2016) The impact of research education on student nurse attitude, skill and uptake of evidence-based practice: a descriptive longitudinal survey. J Clin Nurs 25: 194-203.

27. Malik G, McKenna L, Plummer V (2015) Perceived knowledge, skills, attitude and contextual factors affecting evidence-based practice among nurse educators, clinical coaches and nurse specialists. Int J Nurs Pract 21: 46-57.

28. Ehrenfeld M, Eckerling S (1991) Perceptions and attitudes of registered nurses to research: A comparison with a previous study. J Adv Nurs 16: 224-232.

29. Sanzero Eller L, Kleber E, Lan Wang S (2003) Research knowledge, attitudes and practices of health professionals. Nursing Outlook 51: 165-170.

30. Grant HS, Stuhlmacher A, Bonte-Eley S (2012) Overcoming barriers to research utilization and evidence-based practice among staff nurses. J Nurses Staff Dev 28: 163-165.

31. Hicks C (1995) The shortfall in published research: A study of nurses' research and publication activities. Journal of Advanced Nursing 21: 594-604.

32. Marsh GW, Brown TL (1992) The measurement of nurses' attitudes toward nursing research and the research environment in clinical settings. Journal of Clinical Nursing 1: 315-322.

33. Parahoo K (1998) Research utilization and research related activities of nursing in Northern Ireland. J Psychiatr Ment Health Nurs 31: 89-98.

34. Parahoo K (1999) A comparison of pre-Project 2000 and Project 2000 nurses' perceptions of their research training, research needs and their use of research in clinical areas. Journal of Advanced Nursing 29: 237-245.

35. Poster EC, Betz CL, Randell B (1992) Psychiatric nurses' attitudes toward and involvement in nursing research. Journal of Psychosocial Nursing 30: 26-29.

36. Rizzuto C, Bostrom J, Suter WN, Chenitz WC (1994) Predictors of nurses' involvement in research activities. West $\mathrm{J}$ Nurs Res 16: 193-204.

37. Webb C, MacKenzie J (1993) Where are we now? Research-mindedness in the 1990's. Journal of Clinical Nursing 2: 129-133.

38. Carroll DL, Greenwood R, Lynch KE, et al. (1997) Barriers and facilitators to utilization of nursing research. Clinical Nurse Specialist 11: 207-212.

39. Barta KM (1995) Information-seeking, research utilization, 
Citation: Gagnon L (2018) Navigating Evidence Based Practice: A Guide for Nursing Students. J Nurs Pract 2(2):73-80

and barriers to research utilization of pediatric nurse educators. J Prof Nurs 11: 49-57.

40. Goode CJ (1995) Evaluation of research-based nursing practice. Nurs Clin North Am 30: 421-428.

41. Dunn V, Crichton N, Roe B, et al. (1997) Using research for practice: A UK experience of the Barriers scale. Journal of Advanced Nursing 27: 1203-1210.

42. Funk SG, Champagne MT, Tornquist EM, et al. (1995) Administrators' views on barriers in research utilization. Applied Nursing Research 8: 44-49.

43. Mottola CA (1996) Research utilization and continuing/staff development educator. The Journal of Continuing Education in Nursing 27: 168-175.

44. Lewis SL, Prowant BF, Cooper CL, et al. (1998) Nephrology nurses' perceptions of barriers and facilitators to using research in practice. Nephrology Nursing Journal 25: 397-405.

45. McCaughan D, Thompson C, Cullum N, et al. (2002) Acute care nurses' perceptions of barriers to using research information in clinical decision making. Journal of Advanced Nursing 39: 46-60.

46. Nolan M, Morgan L, Curran M, et al. (1998) Evidence-based care: Can we overcome the barriers? British Journal of Nursing 7: 1273-1278.

47. Parahoo K (2000) Barriers to, and facilitators of, research utilization among nurses in Northern Ireland. J Adv Nurs 31: 89-98.

48. Pettengill MM, Gillies DA, Clark CC (1994) Factors encouraging an discouraging the use of nursing research findings. Journal of Nursing Scholarship 26: 143-147.

49. Restas A (2000) Barriers to using research evidence in nursing practice. J Adv Nurs 31: 599-606.

50. Restas A, Nolan M (1999) Barriers to nurses' use of research: An Australian hospital study. Int J Nurs Stud 36: 335-343.
51. Rutledge DN, Ropka M, Green PE, et al. (1998) Barriers to research utilization for oncology staff nurses and nurse managers/clinical nurse specialists. Oncol Nurs Forum 25: 497-506.

52. Walsh M (1997) Perceptions of barriers to implementing research. Nursing Standard 11: 34-37.

53. Nilsson Kajermo KN, Nordstrom G, Kruesbrant A, et al. (1998) Barriers to and facilitators of research utilization, as perceived by a group of registered nurses in Sweden. J Adv Nurs 27: 798-807.

54. Davidson JE, Brown C (2014) Evaluation of nurse engagement in evidence-based practice. AACN Adv Crit Care 25: 43-55.

55. Brown C, Ecoff L, Kim S, et al. (2010) Multi-institutional study of barriers to research utilization and evidence-based practice among hospital nurses. J Clin Nurs 19: 1944-1951.

56. Storer Brown D (1997) Nursing education and nursing research utilization: Is there aconnection in clinical settings? The Journal of Continuing Education in Nursing 28: 258262.

57. Discenso A, Cullum N, Ciliska D (1998) Implementing evidence-based nursing: Some misconceptions. Evid Based Nurs 1: $38-40$

58. Kitson AL, Rycroft-Malone J, Harvey J, et al. (2008) Evaluating the successful implementation of evidence into practice using the PARiSH framework: Theoretical and practical challenges. Implementation Science 3: 1-12.

59. Mills J, Field J, Cant R (2011) Factors affecting evidence translation for general practice nurses. International Journal of Nursing Practice 17: 455-463.

60. Ramos-Morcillo AJ, Fernández-Salazar S, Ruzafa-Martínez M, et al. (2015) Effectiveness of a brief, Basic evidence-based practice course for clinical nurses. World views Evid Based Nurs 12: 199-207. 